\title{
ON THE MULTIPLICATIVE BEHAVIOR OF REGULAR MATRICES
}

\author{
ROBERT E. ATALLA
}

ABSTRACT. Let $T$ be a bounded linear operator on $C(X), X$ compact $T_{2}$, with $T 1=1$. We define $M_{T}$ to be the subalgebra of $C(X)$ consisting of $g$ such that $T(f g)=T f T g$ for all $f$, and give a characterization of $M_{T}$. We apply the characterization to the multiplicative behavior of regular matrices, considering these as linear operators on $C(\beta N \backslash N)$. We also relate invariance properties of a matrix under suitable mappings of the integers to topological properties of its support set in $\beta N \backslash N$, and give an example of a nonnegative multiplicative matrix whose support set is nowhere dense in $\beta M \backslash N$.

Introduction. Let $T=\left(a_{m n}\right)$ be a regular matrix, $N$ the positive integers, and $c_{0}$ the real functions on $N$ which vanish at infinity. Then $T\left(c_{0}\right) \subset c_{0}$, so $T$ induces in a natural way a linear operator on $C(\beta N \backslash N)$ with norm

$$
\|T\|=\lim \sup (m \rightarrow \infty) \sum_{k}\left|a_{m k}\right| \quad \text { (cf. [1]). }
$$

This leads us to consider general operators on $C(X), X$ compact Hausdorff, with the aim of establishing theorems applicable to classical summability theory.

In the first section we characterize the subalgebra $M_{T}$ of $C(X)$ consisting of $g$ such that $T(f g)=T f T g$ for all $f$, and relate $M_{T}$ to the "support set" $K$ of the operator $T$. In the second section we indicate how some known results on multiplicative summability follow easily from our characterization of $M_{T}$, and give a proof of a known theorem on the topological nature of the support set of a regular matrix. In the third section we show how a known relationship between motions of the integers and regular matrices may be used in the study of the topological nature of support sets. In the fourth section we give an example of a nonnegative regular multiplicative matrix such that

$$
\lim (m \rightarrow \infty) \sup \left\{a_{m k}: k \in N\right\}=0,
$$

and whose support set $K$ in $\beta N \backslash N$ is nowhere dense.

In the sequel, $C^{*}(N)$ will be the bounded real valued functions on $N$. If $f \in C^{*}(N), f^{\prime}$ will be its extension to $\beta N$, and $f^{*}$ the restriction

Received by the editors June 4, 1969 and, in revised form, July 7, 1969 and July 22, 1969.

AMS 1969 subject classifications. Primary 4031, 4725; Secondary 5453, 4625.

Key words and phrases. Regular matrix, $C(X)$, multiplicative matrix method, invariant mean, $\beta N$. 
of $f^{\prime}$ to $\beta N \backslash N$. If $A \subset N, A^{\prime}$ will be the closure of $A$ in $\beta N$, and $A^{*}$ $=A^{\prime} \cap(\beta N \backslash N)$. If $W \subset C^{*}(N), W^{*}=\left\{f^{*}: f \in W\right\}$.

1. The algebra $M_{T}$.

1.1 Definition. Let $X$ be a compact $T_{2}$ space, $T$ a bounded linear operator on $C(X)$ such that $T 1=1$,

$$
\begin{aligned}
M_{T} & =\{g \in C(X): T(f g)=(T f)(T g) \text { for all } f \in C(X)\}, \\
C_{T} & =\{f \in C(X): T f=\text { constant }\} .
\end{aligned}
$$

For each $p \in X$, let $m_{p}$ be the Borel measure representing the functional $f \rightarrow(T f)(p)$, let $K_{p}$ be the support set of $m_{p}$, and $K=$ closure $\cup\left\{K_{p}: p \in X\right\}$. We call $K$ the support set of $T$.

Clearly, $M_{T}$ is a Banach algebra with unity. If $X=\beta N \backslash N$ and $T$ is an operator on $C(X)$ induced by a regular matrix, as mentioned in the Introduction, then $C_{T}$ is essentially the bounded convergence field of $T$.

1.2 ThEOREM. $g \in M_{T}$ iff $g$ is constant on each $K_{p}$. If $M_{T}^{\prime}=$ restrictions to $K$ of $M_{T}$, then $T$ acts as a Banach algebra isometry of $M_{T}^{\prime}$ into $C(X)$.

Proof. If $g=\alpha$ on $L=K_{p}$, then since $1=(T 1)(p)=\int d m_{p}$, we have $(T g)(p)=\int_{L} g d m_{p}=\alpha$. Hence if $f \in C(X), \quad(T f g)(p)=\int_{L} f g d m_{p}$ $=\alpha \int_{L} f d m_{p}=T g(p) T f(p)$. Hence $g \in M_{T}$. Conversely if $(T f g)(p)$ $=(T f)(p)(T g)(p)$ for each $f \in C(X)$, then

$$
0=\int_{L} f g d m_{p}-\int_{L} f(T g)(p) d m_{p}=\int_{L} f(g-T g(p)) d m_{p} .
$$

Thus $g-T g(p) \mid L$ is orthogonal to $C(L)$, whence $g-T g(p)$ is identically zero on $L$.

We have shown that $K_{p} \subset g^{-1}(T g(p))$ for each $g \in M_{T}$ and $p \in X$, so the last assertion is immediate.

1.3 THEOREM. If either $T \geqq 0$, or $K_{p}$ has at most two points for each $p$, then $g \in M_{T}$ iff $T\left(g^{2}\right)=(T g)^{2}$.

Proof. If $g \in M_{T}$, the conclusion is trivial. For the converse, first assume $T \geqq 0$. Then each $m_{p}$ is a positive probability measure, and we have $\int g^{2} d m_{p}=\left(\int g d m_{p}\right)^{2}$. Thus we have equality in the CauchySchwartz inequality for the elements $g$ and 1 of $L^{2}(m)$. Hence 1 and $g$ are linearly dependent in $L^{2}(m)$, and it follows easily that $g$ is constant on $K_{p}$, so 1.2 implies $g \in M_{T}$.

If $K_{p}=\{r, s\}$, then $(T g)(p)=a g(r)+b g(s)$ with $a+b=1$, and it is easy to check that $\left(T g^{2}\right)(p)=T g(p)^{2}$ iff $g(r)=g(s)$. 
1.4 Counterexample. Let $T$ be the operator on $C(\beta N \backslash N)$ induced by the matrix operator

$$
\left(T_{1} f\right)(n)=f(3 n-2)+f(3 n-1)-f(3 n) .
$$

It is easy to see that for each $p \in \beta N \backslash N, K_{p}$ contains three points. Let $f \in C^{*}(N)$ satisfy $f(3 n-1)=f(3 n)$, so $\left(T_{1} f\right)(n)=f(3 n-2)$, and clearly $T_{1} f^{2}=\left(T_{1} f\right)^{2}$, and the same holds for the extension $f^{*}$ of $f$ to $\beta N \backslash N$. Unless $\lim f(n)=0$, the extension of $f$ to $\beta N \backslash N$ will not be constant on each $K_{p}$. For if $K_{p}=\{r, s, t\}$, then we have, say, $f^{*}(s)$ $=-f^{*}(t)$; so if $f^{*}$ is constant on $K_{p}$, then $0=f^{*}(s)=f^{*}(t)=f^{*}(r)$.

Suppose, in addition, that $\alpha=\lim _{n \rightarrow \infty} f(3 n-2)$ exists, so $f^{*} \in C_{T} \backslash M_{T}$. Then $\left(f^{*}\right)^{n} \in C_{T} \backslash M_{T}$ for all $n$, and $T\left(f^{*}\right)^{n}=\alpha^{n}$.

1.5 Theorem. $g \in C_{T} \cap M_{T}$ iff $g \mid K$ is constant. If, further, $T$ satisfies the conditions of 1.3, then the following are equivalent: (a) $C_{T}$ is a Banach algebra, and the restriction of $T$ to $C_{T}$ is multiplicative, (b) $C_{T} \subset M_{T}$, i.e., $C_{T}=\{f \in C(X): f \mid K=$ constant $\}$.

Proof. The first assertion follows from 1.2 and the relevant definitions. That (b) implies (a) in the second assertion is obvious. To show (a) implies (b), suppose $g \in C_{T} \backslash M_{T}$. If $g^{2} \notin C_{T}$, then $C_{T}$ is not an algebra, while if $g^{2} \in C_{T}$, then 1.3 implies $T\left(g^{2}\right) \neq(T g)^{2}$, so $T$ is not multiplicative on $C_{T}$.

1.6 REMARK. Suppose $T$ is an operator on $C(\beta N \backslash N)$ induced by a regular matrix. If the convergence field $C_{T}$ is a Banach algebra and $T$ is multiplicative, then the support set $K$ of Definition 1.1 is the same as the set $F(\phi)$ of the paper of Henriksen [4, p. 432].

1.7 THEOREM. Let $T$ be an operator on $C(\beta N \backslash N)$ induced by a regular matrix. Then the subalgebras $M_{T}$ and $T\left(M_{T}\right)$ are both "large" subalgebras in the following sense: for each nonvoid open $V$ in $\beta N \backslash N$, there exists $g \in M_{T}$ which takes the values 0 and 1 on $V$ and likewise for $T\left(M_{T}\right)$.

Proof. The proof is essentially contained in the paper of G. M. Petersen [7]. The sequences which are derived, as in the lemma of p. 257, from the basic sequence $\left\{u_{k}\right\}$, have extensions to $\beta N \backslash N$ which are elements of $M_{T}$. This is best seen by modifying the computation at the bottom of p. 257 to show that if $r=r\left\{r_{n}\right\}$ is the sequence derived from the $\left\{u_{k}\right\}$, and $s=\left\{s_{n}\right\}$ is any other sequence (it need not be null), then

$$
\lim _{n \rightarrow \infty}\left(\sum_{n} a_{m n} s_{n} r_{n}-\sum_{n} a_{m n} s_{n} \sum_{n} a_{m n} r_{n}\right)=0 .
$$


In the proof of Theorem 2 of that paper it is shown essentially that given a zero set in $\beta N \backslash N$, a suitable choice of zeros and ones in $\left\{u_{k}\right\}$ will give an $\left\{r_{n}\right\}$ whose extension to $\beta N \backslash N$ is in $M_{T}$ and which takes the values 0 and 1 on the zero set; and another suitable choice will give an element of $T\left(M_{T}\right)$ which does the same.

2. Applications to summability. The results of the preceding section lead to alternative proofs of some of the results in the paper [6] of Hill and Sledd. Due to limitations of space we must leave the details to the reader. We point out only that if $|A|$ is as on p. 410 of their paper, then $|A|^{*}=\left(C_{T}\right)^{*} \cap\left(M_{T}\right)^{*}$.

We now give a theorem on the topological nature of the support set of an operator on $C(\beta N \backslash N)$ induced by a positive matrix. This result was announced without proof by Henriksen and Isbell [5], along with several other interesting results. Theorem 4.1 of Hill and Sledd [6] (also announced without proof in [5]) is easily seen to be equivalent to this result. Since a direct proof seems not to exist in print, we give one here. The proof is based on an idea of Rudin [11].

2.1 THEOREM. Let $T=\left(t_{m k}\right)$ be a nonnegative matrix whose columns are null sequences and whose rows are a bounded subset of $l^{1}$, and let $K$ be the support set in $\beta N \backslash N$ of the operator on $C(\beta N \backslash N)$ induced by $T$. If $f \in C(\beta N \backslash N)$ and $f$ vanishes on $K$, then $f$ vanishes on a neighborhood of $K$.

Proof. Let $\mathcal{F}$ be the class of subsets $A$ of $N$ such that $T-$ $\lim \left(1-\chi_{A}\right)=0$, and $A^{*}$ be as in the Introduction. Then $K$ $=\bigcap\left\{A^{*}: A \in \mathcal{F}\right\}$. It is easy to check that it suffices to prove that if $A_{k} \in \mathcal{F}, k=1,2, \cdots$, then there exists $A \in \mathcal{F}$ such that $A^{*} \subset \cap_{k}\left(A_{k}\right)^{*}$, or, equivalently [11], $A \backslash A_{k}$ is finite for $k=1,2, \cdots$.

We assume without loss of generality that for each $m$, the $m$ th row $T_{m}$ of $T$ has finite support ( $t_{m k}=0$ for all but a finite number of $k$ ) [8, p. 82]. Now it is easy to see that $A_{1} \cap \cdots \cap A_{k} \in \mathcal{F}$ for each $k$. Choose $m(1)<m(2)<\cdots$ such that if $g_{k}$ is the characteristic function of $A_{1} \cap \cdots \cap A_{k}$, then $T_{m}\left(1-g_{k}\right)<k^{-1}$ for $m \geqq m(k)$. Choose $n(1)<n(2)<\cdots \quad$ such that support $T_{m} \subset[0, n(k))$ whenever $m \in[m(k), m(k+1))$. Let $B_{k}=A_{1} \cap \cdots \cap A_{k} \cap[0, n(k))$ and $A$ $=\bigcup_{k} B_{k}$. For each $k, A \backslash A_{k}$ is finite. Further if $m \in[m(k), m(k+1))$, then $T_{m}\left(1-g_{k}\right)=T_{m}\left(1-\chi_{B_{k}}\right)$. So

$$
T_{m}\left(1-\chi_{A}\right) \leqq T_{m}\left(1-\chi_{B_{k}}\right)<k^{-1} .
$$

Hence $A \in \mathcal{F}$. 
3. Relation to invariant means. In this section $\pi$ will be a one-one mapping of the positive integers $N$, whose range has finite complement. If $n \neq \pi^{k} n$ for all $k$ and $n, \pi$ is called a motion. $M_{\pi}$ is the set of $\pi$-invariant positive linear functionals on $C^{*}(N)$ such that $\phi(1)=1$, and

$$
V_{\pi}=\left\{f \in C^{*}(N): \phi(f)=\phi^{\prime}(f) \text { for all } \phi, \phi^{\prime} \in M_{\pi}\right\} .
$$

If $T=\left(a_{m n}\right)$ is a regular matrix transform, we say $T$ is $\pi$-invariant if

$$
\lim (m \rightarrow \infty) T(f-f \circ \pi)(m)=0
$$

for each $f \in C^{*}(N)$. This terminology is from the paper of Raimi [10], in which appears the

THEOREM. $T$ is $\pi$-invariant iff

$$
\lim (m \rightarrow \infty) \sum_{k}\left|a_{m k}-a_{m \pi(k)}\right|=0 .
$$

If $\pi$ is a motion, and the above holds, then $V_{x} \subset C_{T}$, where $C_{T}$ is the convergence field of $T$, and $\phi(f)=T-\lim (f)$ for each $\phi \in M_{*}$ whenever $f \in V_{\pi}$.

(The assumption that $\pi$ is a motion is clearly not needed in Raimi's proof of the first sentence.)

We relate the invariance properties of $T$ to the sets $C_{T}$ and $M_{T}$, where $C_{T}$ is the convergence field of $T$ and

$M_{T}=\left\{g \in C^{*}(N): T(f g)(m)-(T f)(m)(T g)(m) \rightarrow 0\right.$ for all $\left.f \in C^{*}(N)\right\}$.

(Note that $\left(C_{T}\right)^{*}$ and $\left(M_{T}\right)^{*}$ then correspond to the induced operator on $C\left(N^{*}\right)$, as in Definition 1.1.) We show in particular that if $T$ is invariant for some motion $\pi$, then $T$ cannot be multiplicative in the sense of Henriksen [4]. Now if $T$ is invariant for a motion $\pi$, then an elementary calculation gives

$$
\lim (m \rightarrow \infty) \sup \left\{\left|a_{m k}\right|: k \in N\right\}=0,
$$

so it is natural to conjecture that under this last condition $T$ cannot be multiplicative. In $\$ 4$ we produce a counterexample to this, namely a positive multiplicative matrix $T$ with $\lim (m \rightarrow \infty) \sup \left\{a_{m k}: k \in N\right\}$ $=0$. We show further that the support set $K$ of $T$ is nowhere dense in $\beta N \backslash N$, hence is not a $G_{\delta}$. This illuminates the question raised by Henriksen $[4$, p. 432] regarding the topological nature of the support set of a multiplicative matrix.

TheOREM 3.1. Suppose $T$ is invariant under the motion $\pi$, and $g \in M_{T}$. Then $g^{*}=g^{*} \circ \pi$ on the set $K$. 
Proof. By Theorem 1.2 if $\left(M_{T}^{\prime}\right)^{*}$ are restrictions of elements of $\left(M_{T}\right)^{*}$ to $K$, then $T$ acts as an isometry of $\left(M_{T}^{\prime}\right)^{*}$. Now the invariance of $T$ under $\pi$ implies $T\left(f^{*}\right)=T\left(f^{*} \circ \pi\right)$ for the induced operator acting on $C(\beta N \backslash N)$, and for $g^{*} \in\left(M_{T}\right)^{*}, T\left(g^{*}\right)=T\left(g^{*} \circ \pi\right)$ implies $g^{*}$ $=g^{*} \circ \pi$ on $K$.

On pp. 430 and 431 of [4], Henriksen notes that a submatrix of the identity is always a multiplicative method on the space of all (bounded and unbounded) sequences, and that according to a result of Mazur and Orlicz, these are the only multiplicative methods. The situation is quite different when we consider only bounded sequences, as the following theorem and the example of $\$ 4$ will show. (Note also example (3.3) of [4].)

Theorem 3.2. Suppose $\lim (m \rightarrow \infty) \sup \left\{\left|a_{m k}\right|: k \in N\right\}=0$. Then $K$ is not an open set in $\beta N \backslash N$.

Proof. Assuming $K$ is open, we shall obtain a contradiction. Since $K$ is open and closed in $\beta N \backslash N$, there is an infinite $L \subset N$ such that $K$ $=L^{*}$. By Theorem 1.7, $\left(M_{T}\right)^{*}$ contains a nonconstant $f$, and we may assume $f=g^{*}$ for some $g \in C^{*}(N)$ which is constantly equal to

$$
2^{-1}\{\lim \sup g(n)+\lim \inf g(n)\}
$$

on the complement of $L$. By regularity of $T$ we may assume (cf. [3]) that there exist sequences $\lambda(1)<\lambda(2)<\cdots$ and $m(1)<m(2)$ $<\ldots$ such that $m \in[m(k), m(k+1))$ implies $a_{m n}=0$ for $n$ $\notin[\lambda(k-1), \lambda(k+1)]$. We now choose $n(1)<n(2)<\cdots$ as follows:

$$
\begin{aligned}
\lim (k \rightarrow \infty) g(n(2 k)) & =\lim \sup g(n), \\
\lim (k \rightarrow \infty) g(n(2 k+1)) & =\lim \inf g(n),
\end{aligned}
$$

and an interval $[\lambda(k-1), \lambda(k+1)]$ contains at most two elements of the sequence $\{n(k)\}$. Define $\pi \mid N \rightarrow N$ by $\pi(n(k))=n(k+1)$ and $\pi=$ identity on the complement of $\{n(k)\}$. Then clearly $f \circ \pi \neq f$ on $K$. We shall show that $T$ is invariant under $\pi$, obtaining a contradiction to Theorem 3.1. Since $\{n(k)\}$ intersects $[\lambda(k-1), \lambda(k+1)]$ at most twice, if $m \in[m(k), m(k+1)]$ we have

$$
\sum_{k}\left|a_{m k}-a_{m \pi k}\right| \leqq 4 \sup \left\{\left|a_{m k}\right|: k \in N\right\} .
$$

But by hypothesis the right side goes to zero as $m \rightarrow \infty$.

THEOREM 3.3. If $T$ is nonnegative, and is invariant under some motion $\pi$ then the support set $K$ of $T$ is nowhere dense in $\beta N \backslash N$. 
Proof. By the theorem of Raimi cited above, $V_{\pi} \subset C_{T}$. If $A \subset N$ is a set of $\pi$-density $1[9,1.7]$, then $T-\lim \chi_{A}=1$. Let $f \in C(\beta N \backslash N)$ be the characteristic function of $A^{*}$. Then for each $p \in \beta N \backslash N, 1$ $=(T f)(p)=\int f d m_{p}$, so $K_{p} \subset A^{*}$. Hence $K \subset A^{*}$. By Definition 2.3 and Theorem 2.7 of [9] the intersection of all such $A^{*}$ is nowhere dense in $\beta N \backslash N$, hence so is $K$.

TheOREM 3.4. Suppose $T$ is invariant under a motion $\pi$. Then $T$ is not multiplicative.

Proof. Again, $V_{\pi} \subset C_{T}$, and it suffices to produce a $g \in V$ with $\phi(g)=0, \phi\left(g^{2}\right)=1$ for all $\phi \in M_{\pi}$. By Corollary 4.9 of [2] we may assume $\pi$ is a "half-cycle", i.e., there is a $p$ such that the complement of $\left\{p, \pi p, \pi^{2} p, \cdots\right\}$ is finite. We define $g\left(\pi^{k} p\right)$ to be 1 if $k$ is even, -1 if $k$ is odd, and let $g$ be 1 elsewhere. By Lemma 6 of [10], $\left\{p, \pi^{2} p, \pi^{4} p, \cdots\right\}$ and $\left\{\pi p, \pi^{3} p, \pi^{5} p, \cdots\right\}$ each have $\pi$-density $1 / 2$, and it follows easily that $\phi(g)=0$ for each $\phi \in M_{\pi}$. Since $g^{2}=1$ we have $\phi\left(g^{2}\right)=1$.

\section{The example. We will need the}

Lemma. If $T$ is nonnegative, then $T$ is multiplicative iff $\lim T g=0$ implies $\lim T g^{2}=0$.

Proof. In view of Theorem 1.5 it suffices to show that for the induced operator on $C\left(N^{*}\right),\left(C_{T}\right)^{*} \subset\left(M_{T}\right)^{*}$ iff $T g^{*}=0$ implies $T\left(g^{*}\right)^{2}$ $=0$. If $\left(C_{T}\right)^{*} \subset\left(M_{T}\right)^{*}$, the desired conclusion follows from 1.3. Conversely if the condition is satisfied, let $f \in C_{T}$, so that, say, $T f^{*}=k$. Let $g^{*}=f^{*}-k$. Then $T g^{*}=0$, so $0=T\left(g^{*}\right)^{2}=T\left(f^{*}\right)^{2}-2 k T f^{*}+k^{2}$ $=T\left(f^{*}\right)^{2}-k^{2}$, or $T\left(f^{*}\right)^{2}=\left(T\left(f^{*}\right)\right)^{2}$ for any $f \in C_{T}$. Now if $f, g \in C_{T}$, write $f g=4^{-1}\left((f+g)^{2}-(f-g)^{2}\right)$, and by linearity find $T\left(f^{*} g^{*}\right)$ $=T\left(f^{*}\right) T\left(g^{*}\right)$.

We now construct our example of a nonnegative regular matrix which is multiplicative, and such that $\lim (m \rightarrow \infty) \sup \left\{a_{m k}: k \in N\right\}$ $=0 . T$ will have the form

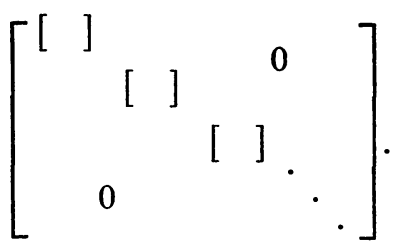

Each row of the $n$th block will have length $2 n$. Half of the terms are $n^{-1}$ and half are 0 . For each possible ordering of these $n^{-1}$ 's and 0 's, 
the $n$th block will contain a row ordered just this way. This matrix was designed so as not to be invariant under any motion. If $\pi$ is a motion, then clearly each block contains a row with

$$
\sum_{k}\left|a_{m k}-a_{m \pi(k)}\right|=2 .
$$

Suppose $\lim (m \rightarrow \infty) \sum_{k} a_{m k} g(k)=0$. We will prove

$$
\lim (m \rightarrow \infty) \sum_{k} a_{m k} g(k)^{2}=0 .
$$

We may assume $-1 \leqq g \leqq 1$. Let $\epsilon>0$, and suppose $n$ is so large that $\left|\sum_{k} a_{m k} g(k)\right|<\epsilon$ on the $n$th block. There exists $L$ such that on this block $a_{m k}=0$ if $k \notin[L+1, L+2 n]$. Let us assume that at least half the numbers $\{g(L+1), g(L+2), \cdots, g(L+2 n)\}$ are nonnegative. (The alternative assumption leads to a similar proof.) If $g(L+k(1)), \cdots, g(L+k(n))$ are any $n$ nonnegative terms, then by using an appropriate row of the block we get (since $-1 \leqq g \leqq 1$ )

$$
\begin{aligned}
0 & <n^{-1} \sum\left\{g(L+k(j))^{2}: j=1, \cdots, n\right\} \\
& \leqq n^{-1} \sum\{g(L+k(j)): j=1, \cdots, n\}<\epsilon .
\end{aligned}
$$

Nowifor an arbitrary row in the block the corresponding sum satisfies

$$
-\epsilon<n^{-1} \sum\{g(1+k(j)): j=1, \cdots, n\}<\epsilon .
$$

Let $\sum^{\prime} g$ and $\sum^{\prime \prime} g$ correspond to the nonnegative and the negative terms of the sum. Then $\left(^{*}\right)$ implies $0 \leqq n^{-1} \sum^{\prime} g^{2} \leqq n^{-1} \sum^{\prime} g<\epsilon$. (The positive terms can be extended to a set of $n$ positive terms, and then $\left.{ }^{*}\right)$ applies.) Together with $\left({ }^{* *}\right)$ this readily gives $n^{-1} \sum^{\prime \prime} g>-2 \epsilon$, or

$$
2 \epsilon>n^{-1} \sum^{\prime \prime}(-g) \geqq n^{-1} \sum^{\prime \prime}(-g)^{2}=n^{-1} \sum^{\prime \prime} g^{2} .
$$

We now have

$$
n^{-1} \sum g^{2}=n^{-1} \sum^{\prime} g^{2}+n^{-1} \sum^{\prime \prime} g^{2}<3 \epsilon .
$$

It follows that $\lim (m \rightarrow \infty) \sum_{k} a_{m k} g(k)^{2}=0$, and the proof is complete.

To show the support set $K$ of $T$ is nowhere dense we first form a new matrix $T_{1}$ as follows: $T_{1}$ will consist of the same blocks as $T$ does, but each entry of the $n$th block will be the number $(2 n)^{-1}$. If $f \geqq 0$, then $2 T_{1}(f) \geqq T(f) \geqq 0$, so that if $K_{1}$ is the support set of $T_{1}$, then $K \subset K_{1}$. But it is easy to see that $T_{1}$ is invariant under the motion $\pi(n)=n+1$. By Theorem 3.3, $K_{1}$ is nowhere dense, hence so is $K$. 
5. Some questions. (a) It may be true that $K$ is nowhere dense whenever $\lim (m \rightarrow \infty) \sup \left\{\left|a_{m k}\right|: k \in N\right\}=0$, but I have not been able to show this.

(b) If $T$ is $\pi$-invariant, then $V_{\pi} \subset C_{T}$, so that the "size" of $V_{\pi}$ gives information about that of $C_{T}$. For instance does $V_{\pi}$ ever separate points of $\beta N \backslash N$ ? (Note that if $T$ is multiplicative, then $C_{T}$ consists only of functions constant on the infinite closed set $K$, so that $C_{T}$ is small in a natural sense.)

(c) Is every infinite closed subset of $\beta N \backslash N$ with the property stated in Theorem 2.1 the support set of a regular matrix?

I have learned that question (b) has been answered in the affirmative by Raimi, and the solution may soon be published. Thus if $T$ is invariant under some motion, then $C_{T}$ separates points of $\beta N \backslash N$. The question of whether this is the case if $T$ is nonmultiplicative but noninvariant remains open.

6. Final remarks. The ideas in $\$ 1$ actually arose from an analysis of Petersen's paper [7]. A closely related idea is that of "aping sequences" due to Erdös and Piranian [3].

I am grateful to the referee for numerous suggestions regarding style, as well as for a simplification in the proof of the lemma at the beginning of $\$ 4$.

AdDED IN PROOF. I have learned that the answer to question (a) is affirmative and easy. R. A. Raimi informs me that question (b) is also answered by S. P. Lloyd, Illinois J. Math. 14 (1970), p. 266. I have been able to answer (c) in the negative; see Notices Amer. Math. Soc., October, 1970.

\section{REFERENCES}

1. R. Atalla and J. Bustoz, On sequential cores and a theorem of R. R. Phelps, Proc. Amer. Math. Soc. 21 (1969), 36-42.

2. D. Dean and R. A. Riami, Permutations with comparable sets of invariant means, Duke Math. J. 27(1969), 467-480.

3. P. Erdös and G. Piranian, The topologization of a sequence space by Toeplitz matrices, Michigan Math. J. 5(1958), 139-148. MR 21 \#812.

4. M. Henriksen, Multiplicative summability methods and the Stone-Cech compactification, Math. Z. 71(1959), 427-435. MR 21 \#7434.

5. M. Henriksen and J. R. Isbell, Multiplicative summability methods and the StoneČech compactification. II, Notices Amer. Math. Soc. 11 (1964), 90-91. Abstract \#608116.

6. J. D. Hill and W. T. Sledd, Approximation in bounded summability fields, Canad. J. Math. 20 (1968), 410-415. MR 36 \#5561.

7. G. M. Petersen, Summability and bounded sequences, Proc. Cambridge Philos. Soc. 55(1959), 257-261. MR 22 \#153. 
8. - Regular matrix transformations, McGraw-Hill, New York and London, 1966. MR 37 \#642.

9. R. A. Raimi, Homeomorphisms and invariant measures for $\beta N-N$, Duke Math. J. 33(1966), 1-12. MR 33 \#6608.

10. - Invariant means and invariant matrix methods of summability, Duke Math. J. 30 (1963), 81-94. MR 27 \#3965.

11. W. Rudin, Homogeneity problems in the theory of Čech compactifications, Duke Math. J. 23(1956), 409-419. MR 18, 324.

Ohio University, Athens, OHio 45701. 